\title{
The Role of Biology and Culture in Veblenian Consumption Dynamics
}

\author{
Christian Cordes* \\ Max Planck Institute of Economics \\ Evolutionary Economics Group
}

August 2007

\begin{abstract}
This paper incorporates aspects of humans' evolved cognition into a formal model of cultural evolution and scrutinizes their interactions with population-level processes. It is shown how the biased transmission of different kinds of behavior via cultural learning processes influences agents' consumption behavior. Thereby, the model's learning dynamics are capable of generating typical Veblenian consumption dynamics. In addition, this paper scrutinizes on the role of humans' biological heritage and Darwinian concepts in the development of economic theories in general. Moreover, the relation of the ontological basis of biological and cultural evolution is addressed.
\end{abstract}

Key words: Conspicuous consumption - Economic theory development - Evolutionary economics - Darwinism - Cultural evolution

JEL Classification: B41, B52, A12, D11, C60

\footnotetext{
*Address: Kahlaische Str. 10, 07745 Jena, Germany; e-mail: cordes@econ.mpg.de, phone: +49(0)3641/686832, fax: $+49(0) 3641 / 686868)$.
} 


\section{Introduction}

“...it is necessary, in order to his own peace of mind, that an individual should possess as large a portion of goods as others with whom he is accustomed to class himself; and it is extremely gratifying to possess something more than others." (Veblen, 1899, 31)

In his book "The Theory of the Leisure Class" (1899), Thorstein Veblen posed the question whether there is a level of economic development that will satisfy people's wants. According to him, human consumption behavior essentially is motivated by two forces: (1) some of it is driven by the desire to meet basic wants and to make life easier and (2) a large proportion of it is driven by the desire to gain and maintain status and meeting other people's expectations. As Veblen put it, the utility agents receive from consumption goods is as much determined by the social signals as it is by meeting basic wants: "The tendency in any case is constantly to make the present pecuniary standard the point of departure for a fresh increase of wealth; and this in turn gives rise to a new standard of sufficiency and a new pecuniary classification of one's self as compared with one's neighbours" $(1899,31)$. Starting from these observations, he concluded that "...no general increase of the community's wealth can make any approach to satiating this need, the ground of which is the desire of every one to excel every one else in the accumulation of goods" $(1899,32)$.

Veblen was convinced that much of what we buy is driven by social convention and does not meet a biological need: "If, as is sometimes assumed, the incentive to accumulation were the want of subsistence or of physical comfort, then the aggregate economic wants of a community might conceivably be satisfied at some point in the advance of industrial efficiency; but since the struggle is substantially a race for reputability on the basis of an invidious comparison, no approach to a definite attainment is possible" $(1899,32)$. If people's wants were determined by an absolute standard, then an increasing productivity would allow us to work less. However, this is not what we observe. The reason is that agents' wants are continually increasing by means of individual and social learning (Witt, 2001). As agents observe others increasing their consumption expenditures, they feel obliged to do the same. According to Veblen, conspicuous consumption establishes standards regarding what consumption patterns agents strive for. Increases in productivity obviously raise the socially expected standards of consumption. Veblen's argument is that humans are instinctively concerned about their position and status within their group.

In addition, Veblen's works figure prominently in an ongoing conceptual-methodological debate in evolutionary economics on the general role of biological, Darwinian concepts, metaphors, and analogies in economic theory development. While evolutionary principles are applicable to almost any dynamical system, many researchers in the social sciences approach cultural evolution via an analogy with biological evolution (e.g., Campbell, 1965; Nelson and Winter, 1982; Hodgson, 2002; Hodgson and Knudsen, 2006; Knudsen, 2002; Becker et al., 2005). These avenues argue that evolutionary aspects of the biological and the cultural spheres both involve the general Darwinian principles of variation, inheritance, and selection. There is some debate going on whether such an analogy construction is helpful to further theory development in economics (e.g., Cordes, 2006, 2007b; Hodgson, 2002; Vromen, 2004; Witt, 2004). If this analogy is expected to hold, then it must be able to explain concrete phenomena in the economic domain and their underlying causal mechanisms. We argue here that thinking too narrowly in terms of some abstract principles derived form a generalized Darwinism impedes theoretical progress in the social sciences. As an alternative, we propose the application of domain-specific models of cultural evolution that take into account humans' evolved social learning capacities. 
For this purpose, a model of cultural evolution that formalizes Veblen's approach to conspicuous consumption and its underlying dynamics is offered. Formal models of cultural evolution analyze how cognitive processes of human agents combine with patterns of social interaction to generate the distributions and dynamics of cultural variants, for example, different consumption behaviors, in a population (Henrich and Boyd, 2002). We will argue that the parallels between biological evolution and Veblen's views on cultural evolution are limited. In fact, biological concepts contributed very little to his views on cultural evolution (see Jennings and Waller, 1998; Cordes, 2007b): Veblen made no use of Darwinian concepts to describe the causal mechanisms of cultural evolution; his references to biological evolution in this context are methodological and metaphorical. Biological arguments, however, are nevertheless pivotal to Veblen's concept of socio-economic change for they account for the cognitive setup of humans that provides the foundations on which cultural evolution rests - an ontological continuity between these two domains (see Witt, 2003). The model addresses concerns arising from the biology-culture analogy by demonstrating that cultural evolution deviates substantially from the biological analogy, while nevertheless producing evolutionary dynamics. Consequently, we will scrutinize whether an application of causal mechanisms derived from Darwinian concepts of biological evolution to the social sciences is warranted.

The paper proceeds as follows. In Section 2, we present a model of cultural transmission and consumption behavior in which individuals are characterized by interacting multiple cultural traits. We will see how a prestige-biased force can lead to a "runaway" process in cultural learning. Next, Section 3 discusses - based on the findings of the model - the cultural and biological determinants of human consumption behavior. In this context, focus is put on the differing causal mechanisms underlying cultural and biological evolution. Section 4 concludes the paper.

\section{A model of cultural transmission and consumption behavior}

"...the propensity for emulation is probably the strongest and most alert and persistent of the economic motives proper" (Veblen, 1899, 110).

Formal models of cultural evolution analyze how cognitive and affective processes combine with patterns of social interaction to generate the distributions and dynamics of cultural variants, for example, consumption behaviors (Henrich and Boyd, 2002). Thereby, social learning is the basis of human culture. These learning capabilities were crafted by natural selection, are speciesspecific, and are constrained and biased in many ways (e.g., Boyd and Richerson, 1985; Rogers, 1988; Tomasello, 1999; Singer, 2000). For culture depends on processes of social learning, population-based dynamic concepts are useful for understanding how such processes work. As will be shown in the following, such a concept is suitable to depict Veblenian consumption dynamics.

Suppose that people tend to imitate the consumption patterns of prestigious people and that prestige is indicated by certain status goods. We will call such a consumption pattern the "indicator trait" of a cultural role model in the following. This process will cause more people to consume these status goods. This is what Veblen (1899) called "conspicuous consumption". Moreover, it will also increase the propensity to imitate the prestigious agents: people also acquire beliefs from the prestigious agents about who should be imitated, and the most prestigious people will prefer more prestige - indicated by the consumption of status goods than the population as a whole. This is captured by what we will denote as "preference trait". A preference trait affects which cultural variants of the indicator trait an individual finds attractive. 
The resulting dynamic is a cultural "runaway" process that feeds back on itself (see Richerson and Boyd, 2005, 164). As Veblen put it: "In order to stand well in the eyes of the community, it is necessary to come up to a certain, somewhat indefinite, conventional standard of wealth; ... and anything in excess of this normal amount is meritorious" $(1899,30)$.

As a result, an exaggeration of cultural variants, for example, certain consumption patters, signaling status in human societies can be observed. Richerson and Boyd $(2005,164)$ provide a nice example for such a prestige-biased force that causes a "runaway" cultural dynamic:

"For example, on the island of Ponpae in the Pacific, a man's prestige is partly determined by his contribution of very large yams to periodic feasts. Prize yams require up to a dozen men to carry, and their cultivation is inefficient from the point of view of food production. We imagine an evolutionary scenario in which, at the beginning, people just brought their best produce to the feast, and the size and number of yams were straight-forward indicators of farming ability. Then, as the idea that the best people would contribute the biggest yams took hold, families began to devote special effort to grow big yams, and the custom of growing giant yams took off. In California, where we live, the twelve-man yam comes to mind when we see a Hummer II rolling down a Los Angels boulevard."

The theory of cultural evolution contains a classification of different modes or mechanisms of cultural transmission (Cavalli-Sforza and Feldman, 1981, 54ff): vertical transmission is used to denote transmission from parent to offspring, while horizontal transmission describes the passing on of cultural traits between members of the same generation. In addition, oblique transmission stands for the transmission from a member of a given generation to a member of the next (or later) generation who is not her child or direct descendant. Moreover, cultural transmission is biased; people tend to acquire some cultural variants rather than others. We assume two biases here: a role model bias and a direct bias. First, the choice of a cultural trait can be based on the observable attributes of the individuals who exhibit the trait (Richerson and Boyd, 2005, 69; Harrington Jr., 1999). Such a model-based bias includes a predisposition to imitate successful or prestigious individuals. ${ }^{1}$ In general, such an indirect bias results if social learners use the value of a second character that characterizes a model (e.g., prestige or status) to determine the attractiveness of that individual as a model for the primary character (e.g., a certain consumption behavior). Second, individuals are more likely to adopt some cultural variants based on their content (Boyd and Richerson, 1985, 135; Richerson and Boyd, 2005, 69). Such a content-based or direct bias can result from the calculation of costs and benefits associated with alternative variants or from cognitive structures that cause people to preferentially adopt some cultural variants rather than others. ${ }^{2}$ In general, a cultural transmission rule is characterized by direct bias if one cultural variant is more attractive than others. A directly biased transmission creates a force that increases the frequency of the culturally transmitted variant that is favored by the bias. Veblen's instinct-based human concern about status within a group and the resulting preference for status-signaling goods is an example for such a content-based bias.

To formally depict these facets of cultural evolution, this section draws on a model of cultural transmission devised by Boyd and Richerson (1985, ch. 8). To model a process that can cause the indicator trait, for example, a certain consumption pattern or consumption level, to coevolve with the preference trait, for example, a certain notion of what is an appropriate level of consumption

\footnotetext{
${ }^{1}$ It is not necessary that all people consciously emulate the wealthy and prestigious agents. Many people simply try to live up to the standards set by the consumption patterns of these actors to avoid violating conventions. Referring to those individuals who adopt the cultural variants of the majority, Veblen wrote: "On pain of forfeiting their good name and their self-respect in case of failure, they must conform to the accepted code, at least in appearance" (1899, $84)$.

${ }^{2}$ This process can be unconscious. See for an example of such a bias Cordes (2005).
} 
to indicate status, so as to cause the indicator trait to take on exaggerated forms, we derive recursion equations for the mean values of the indicator and the preference traits in a population. We will then determine the effects of direct and indirect biased transmission on the joint distribution of these two traits in the population. First, naïve individuals acquire both the indicator trait and the preference trait via unbiased vertical transmission. Next, during an episode of oblique transmission, these two traits are affected by directly and indirectly biased cultural transmission. In a final stage, we look at the probability that an individual characterized by a certain value of her indicator trait becomes a cultural role model in a certain environment. The model assumes that cultural traits are non-discrete and that naïve individuals sample $n$ cultural role models and adopt a weighted average of their observed behavior, i.e., this is a process of "blended inheritance".

We describe the blending of two quantitative characters via cultural transmission. We begin with the effects of oblique transmission as defined above. Let $\bar{X}_{I}$ and $\bar{X}_{P}$ be the means of the indicator and the preference traits in the population after vertical transmission just before oblique transmission takes place. Moreover, we assume that each naïve individual is exposed to $n$ role models $\left(Z_{I 1}, \ldots, Z_{I n}\right)$ and is characterized by the preference trait value, $X_{P O}$, and the indicator trait, $X_{I O}$. Her estimate of the $j$ th model's indicator trait is given by $Z_{I j}$. The transmission of the indicator trait is subject to direct bias, i.e., the consumption of status goods is inherently attractive, as Veblen put it. This means that one part of the weight of the $j$ th role model is a function of the indicator trait, $Z_{l j}$, and the value of the preference trait in the naïve individual, $X_{P O}: \beta\left(Z_{I j}, X_{P O}\right)$. In total, the importance of the $j$ th model in the transmission of the indicator trait is given by a basic weight, $\alpha_{I j}$, that depends on the $j$ th model's social role and the direct bias function, $\beta(\bullet)$. Finally, the weights are normalized by the denominator so that they give the weight of the $j$ th model relative to the other models encountered by the individual in question. We assume that the value of the indicator trait after oblique transmission, $X_{I O}^{\prime}$, is given by the following blending rule:

$$
X_{I O}^{\prime}=\frac{\sum_{j=1}^{n} Z_{I j} \alpha_{I j}\left(1+\beta\left(Z_{I j}, X_{P O}\right)\right)}{\sum_{j=1}^{n} \alpha_{I j}\left(1+\beta\left(Z_{I j}, X_{P O}\right)\right)},
$$

where the individual's estimated value of $Z_{I j}$ of the $j$ th model is calculated as above. The preference trait affects which variants of the indicator trait an individual finds attractive. This does not imply, however, that these choices are always conscious. It is sufficient that agents tend disproportionately to imitate cultural role models with some variants of the indicator trait.

Furthermore, to represent the indirectly biased preference trait, $X_{P O}$, we again assume that the weight of the $j$ th model is a function of the indicator trait's value, $Z_{I j}$, and the value of the preference trait in the naïve individual, $X_{P O}: \Theta\left(Z_{I j}, X_{P O}\right)$. Two naïve individuals exposed to the same set of cultural models will, on average, adopt different cultural variants of traits affected by the role model bias. Below, we will assume that the form of the indirect bias function, $\Theta(\bullet)$, is generally similar to that of the direct bias function, $\beta(\bullet)$, but that the strengths of the two 
processes may be different. Again, the importance of the $j$ th model in the transmission of the indirectly biased trait also depends on a basic weight, $\alpha_{P j}$, that may be different from the other basic weight above. In a similar manner, $\alpha_{P j}$ is modified by function $\Theta(\bullet)$, the indirect bias function. $Z_{P j}$ denotes the naïve individual's estimate of the $j$ th model's preference trait $\left(Z_{P 1}, \ldots, Z_{P n}\right)$. Then, the value of the preference trait after oblique transmission is given by

$$
X_{P O}^{\prime}=\frac{\sum_{j=1}^{n} Z_{P j} \alpha_{P j}\left(1+\Theta\left(Z_{I j}, X_{P O}\right)\right)}{\sum_{j=1}^{n} \alpha_{P j}\left(1+\Theta\left(Z_{I j}, X_{P O}\right)\right)} .
$$

Here, the value of the indicator trait that maximizes $\Theta(\bullet)$ is the most attractive variant of the indicator trait. Individuals that exhibit this value will, on average, have the greatest influence in the transmission of the indirectly biased cultural variant, in this case the preference trait. Here, social learners use the value of a second character - the indicator trait - that characterizes a model to determine the attractiveness of that individual as a model for the primary character - the preference trait.

Given a joint distribution of the two cultural variants that is approximately bivariate normal, it is sufficient to keep track of the means and variances of each of the characters and their covariance. ${ }^{1}$ Moreover, given the models assumptions, the variances and the covariances reach a stable equilibrium independent of the values of the means, i.e., the variances are at equilibrium at values $V_{I}$ and $V_{P}$, while the covariance is at an equilibrium value $C$. Therefore, for we want to calculate the distribution of $X_{I}$ in the population after cultural transmission, $P\left(X_{I}^{\prime}\right)$, given the distribution before transmission, $P\left(X_{I}\right)$, we derive recursions for the mean values of the indicator and preference traits. Due to the fact that we assume $P\left(X_{I}\right)$ to be normally distributed, we only need to deduce the effects of transmission on the mean of $P\left(X_{I}\right)$. Then, the mean value of the indicator trait, $X_{I}$, in the population after transmission, $\bar{X}_{I}^{\prime}$, is

$$
\bar{X}_{I}^{\prime}=\bar{X}_{I}+\left(1-\sum_{j=1}^{n} \alpha_{I j}^{2}\right) \operatorname{Cov}\left(Z_{I}, \beta\left(Z_{I}, X_{P O}\right)\right) .^{2}
$$

Furthermore, we assume that (1) sets of cultural role models are formed at random and (2) the effects of biases in any single learning step are small.

Accordingly, the mean value of the preference trait after oblique transmission is given by the expression

$$
\bar{X}_{P}^{\prime}=\bar{X}_{P}+\left(1-\sum_{j=1}^{n} \alpha_{P j}^{2}\right) \operatorname{Cov}\left(Z_{P}, \Theta\left(Z_{I}, X_{P O}\right)\right) .
$$

\footnotetext{
${ }^{1}$ The variances and covariances refer to the population of models who participate in oblique transmission.

${ }^{2}$ For a detailed derivation see Boyd and Richerson (1985, 141-144).
} 
In order to facilitate an evaluation of the covariance terms above, we need to define a particular functional form for the direct and indirect bias expressions. Moreover, the definition of the cultural transmission rules requires that the direct bias function, $\beta\left(Z_{I j}, X_{P O}\right)$, be chosen so that $1+\beta\left(Z_{I j}, X_{P O}\right)$ is greater than zero for all possible values of $Z_{I j}$ and $X_{P O}$. One plausible form of $\beta(\bullet)$ that satisfies these conditions is a Gaussian bias function

$$
\beta\left(Z_{I j}, X_{P O}\right)=b \exp \left(\frac{-\left(Z_{I j}-X_{P O}\right)^{2}}{2 B}\right),
$$

where $b<<1$. The indirect bias function has the similar form (whereby $u<<1$ )

$$
\Theta\left(Z_{I j}, X_{P O}\right)=u \exp \left(\frac{-\left(Z_{I j}-X_{P O}\right)^{2}}{2 \theta}\right) .
$$

The intensity of the direct bias acting on the indicator trait is measured by $1 / B$. Similarly, the intensity of indirect bias acting on the preference trait is given by $1 / \theta$. If $B$ and $\theta$ are small, then small differences in observed behavior among role models can have a large effect on the probability that a model is imitated and vice versa.

Equations (5) and (6) say that, given the choice between cultural parents with different cultural variants, naïve individuals will tend to imitate the cultural role model whose observed behavior is closest to the value $X_{P O}$. In this context, the value of the preference trait in the naïve individual, $X_{P O}$, determines which value of the indicator trait maximizes the weight of a role model in oblique transmission of both the traits. If both biases are weak, i.e., $B, \theta>>V_{I}, C$, then it can be shown that the means after transmission are

$$
\begin{aligned}
& \bar{X}_{I}^{\prime}=\bar{X}_{I}+V_{I} B^{\prime}\left(\bar{X}_{P}-\bar{X}_{I}\right) \\
& \bar{X}_{P}^{\prime}=\bar{X}_{P}+C \theta^{\prime}\left(\bar{X}_{P}-\bar{X}_{I}\right)
\end{aligned}
$$

where the parameter $B^{\prime}$ with

$$
B^{\prime}=\left(1-\sum_{j=1}^{n} \alpha_{I j}^{2}\right)\left(\frac{b}{B}\right)
$$

measures the effective intensity of directly biased transmission acting on the indicator trait. In addition, the parameter $\theta^{\prime}$ measures the effective intensity of indirect bias acting on the preference trait:

$$
\theta^{\prime}=\left(1-\sum_{j=1}^{n} \alpha_{P j}^{2}\right)\left(\frac{u}{\theta}\right)
$$


In a next step, we specify the probability that an individual characterized by the cultural variant $X_{I}$ becomes a cultural role model in a particular environment $H$. This enables us to later analyze something akin to what proponents of a generalized Darwinism call "selection environment" and the problems connected to this notion in the cultural sphere. We assume that this probability, $W\left(X_{I}\right)$, has the following Gaussian form:

$$
W\left(X_{I}\right)=\exp \left(\frac{-\left(X_{I}-H\right)^{2}}{2 S}\right)
$$

In the case of such a Gaussian function, the ordinate measures the influence of individuals in cultural transmission as a function of their cultural variant, $X_{I}$. The intensity of this influence is measured by the parameter $S$, while the strongest influence in environment $H$ is given by the value $H$. The latter implies that individuals tend to imitate the cultural role models whose behavior is closest to the value $H$. Furthermore, if $S$ is small, then small differences in the value of $X_{I}$ have a large effect on the probability that a model is imitated. On the other hand, if $S$ is large, then differences in observed behavior among models will have only a small effect on the probability that their cultural variant is adopted. If the intensity of this effect, denoted by $S$, is small compared to $V_{I}$ (i.e., $\left.S>>V_{I}\right)$, then $W\left(X_{I}\right)$ is approximately

$$
W\left(X_{I}\right)=1-\frac{\left(X_{I}-H\right)^{2}}{2 S}
$$

Given these assumptions, the mean values of the two traits in the population are

$$
\begin{aligned}
& \bar{X}_{I}^{\prime \prime}=\bar{X}_{I}+V_{I}\left(B^{\prime}\left(\bar{X}_{P}-\bar{X}_{I}\right)+\left(\frac{H-\bar{X}_{I}}{S}\right)\right) \text { and } \\
& \bar{X}_{P}^{\prime \prime}=\bar{X}_{P}+C\left(\theta^{\prime}\left(\bar{X}_{P}-\bar{X}_{I}\right)+\left(\frac{H-\bar{X}_{I}}{S}\right)\right) .
\end{aligned}
$$

It is now possible to calculate the equilibrium values for $\bar{X}_{I}$ and $\bar{X}_{P}$ : at equilibrium these values do not change so $\bar{X}_{I}^{\prime \prime}-\bar{X}_{I}=0$ and $\bar{X}_{P}^{\prime \prime}-\bar{X}_{P}=0$. Considering this gives us two coupled recursions, one describing the development of $\bar{X}_{I}$ in time (15) and another one for the changing values of $\bar{X}_{P}$ (16):

$$
\Delta \bar{X}_{I}=V_{I}\left(B^{\prime}\left(\bar{X}_{P}-\bar{X}_{I}\right)+\left(\frac{H-\bar{X}_{I}}{S}\right)\right) \quad\left(\bar{X}_{I}^{\prime \prime}-\bar{X}_{I}=\Delta \bar{X}_{I}\right)
$$

\footnotetext{
${ }^{1}$ See Boyd and Richerson $(1985,102)$ for details.
} 


$$
\Delta \bar{X}_{P}=C\left(\theta^{\prime}\left(\bar{X}_{P}-\bar{X}_{I}\right)+\left(\frac{H-\bar{X}_{I}}{S}\right)\right) \quad\left(\bar{X}_{P}^{\prime \prime}-\bar{X}_{P}=\Delta \bar{X}_{P}\right) .
$$

Next, we solve for $\hat{\bar{X}}_{I}$ and $\hat{\bar{X}}_{P}$ denoting the equilibrium values of the indicator and the preference traits. $\Delta \bar{X}_{I}$ becomes zero if

$$
\hat{\bar{X}}_{I}=\frac{H+B^{\prime} S \bar{X}_{P}}{1+B^{\prime} S}
$$

and $\Delta \bar{X}_{P}$ becomes zero if

$$
\hat{\bar{X}}_{P}=\bar{X}_{I}+\frac{\bar{X}_{I}-H}{S \theta^{\prime}} .
$$

By combing $\hat{\bar{X}}_{P}$ and (17) we yield $\hat{\bar{X}}_{I}=H$. Substituting $\hat{\bar{X}}_{I}$ into (18) gives us $\hat{\bar{X}}_{P}=H$. Therefore, if $\hat{\bar{X}}_{I}=\hat{\bar{X}}_{P}=H$, both recursions equal zero and for every value of $H, \hat{\bar{X}}_{P}=\hat{\bar{X}}_{I}$ is an equilibrium of the two-dimensional dynamic system consisting of (15) and (16).

Next, to further analyze the model's properties, we compute the trajectory of the system (see, e.g., McElreath and Boyd, 2007, ch. 8):

$$
\frac{\Delta \bar{X}_{P}}{\Delta \bar{X}_{I}}=\frac{C\left(\theta^{\prime}\left(\bar{X}_{P}-\bar{X}_{I}\right)+\frac{H-\bar{X}_{I}}{S}\right)}{V_{I}\left(B^{\prime}\left(\bar{X}_{P}-\bar{X}_{I}\right)+\frac{H-\bar{X}_{I}}{S}\right)} .
$$

We first consider the case $\frac{H-\bar{X}_{I}}{S}=0$, which implies that we do not differentiate between different strengths of influence of different cultural role models. The parameter $H$ drops out of equation (19) and $\hat{\bar{X}}_{I}=\hat{\bar{X}}_{P}$ is an equilibrium. This is a convenient simplification that does not alter the qualitative results of our analysis. We will later again consider this parameter. Equation (19) then simplifies to

$$
\frac{\Delta \bar{X}_{P}}{\Delta \bar{X}_{I}}=\frac{C \theta^{\prime}}{V_{I} B^{\prime}} .
$$

Given these simplifications, the only forces acting on the learning dynamic are direct and indirect bias. To visualize the dynamics of this model, figure 1 shows a phase plot of its evolutionary trajectories. 

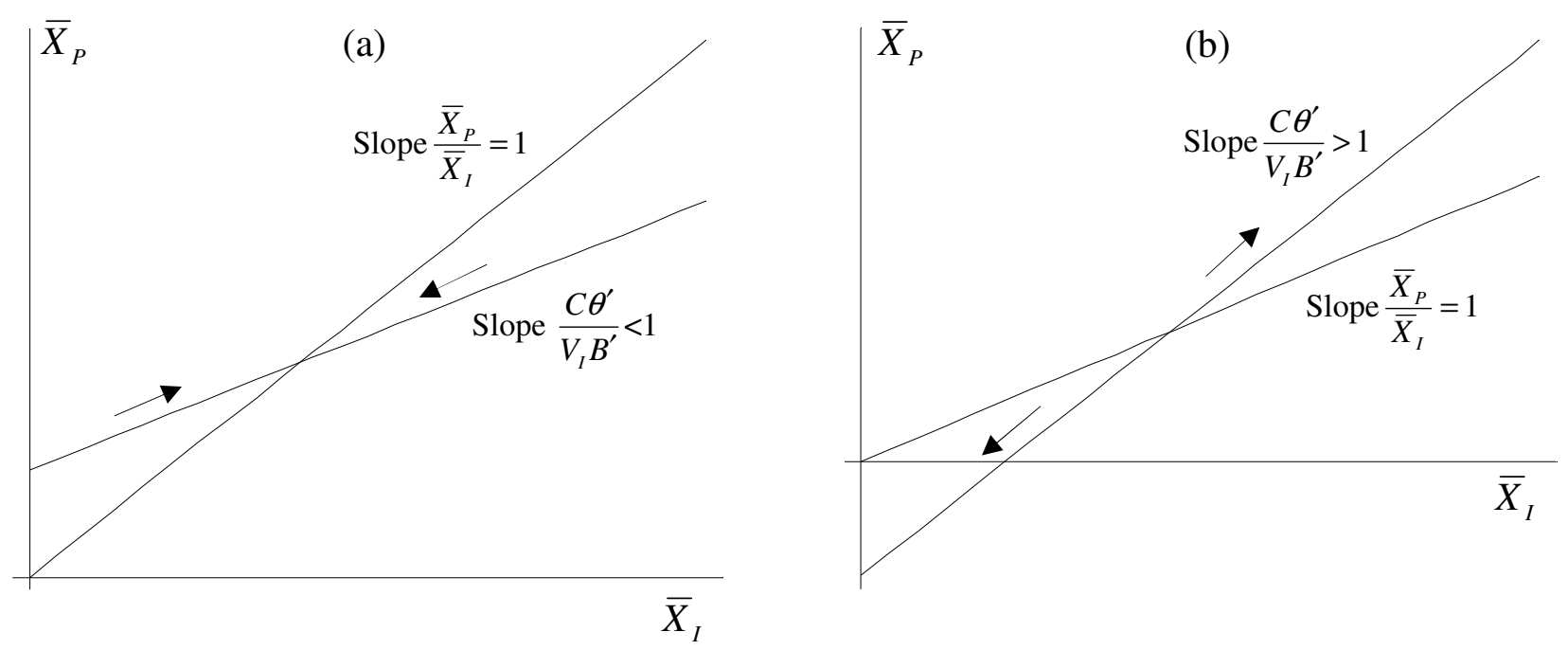

Figure 1: A phase plot of the model's evolutionary trajectories.

If $\bar{X}_{I}$ changes one unit as a result of biased transmission, $\bar{X}_{P}$ changes $\frac{C \theta^{\prime}}{V_{I} B^{\prime}}$ units as a result of the correlated effects. Both, $\bar{X}_{I}$ and $\bar{X}_{P}$ increase above the line of equilibrium, i.e., in this case, the 45-degree line through the origin with slope one. They both decrease below this line of equilibrium. The other lines give the trajectories of groups of social learners that begin at different initial points and have slope $\frac{C \theta^{\prime}}{V_{I} B^{\prime}}$. Given the case depicted in (a), the slope of the trajectory is less than the slope of the line of equilibrium and the system is stable. On the other hand, in case (b), if the trajectory's slope is greater, the population evolves stronger and stronger preference and indicator traits.

If $\theta^{\prime} C>B^{\prime} V_{I}$, i.e., if the indirect bias, $\theta^{\prime}$, is larger than the direct bias effect, $B^{\prime}$, then the mean of the preference trait in the population, $\Delta \bar{X}_{P}$, is increasing faster than the mean of the indicator trait, $\Delta \bar{X}_{I}$, and both traits "run away". As a consequence, the distance of the population means from the line of equilibrium is increasing from one learning step to the next $\left(\bar{X}_{P}^{\prime}-\bar{X}_{I}^{\prime} \geq \bar{X}_{P}-\bar{X}_{I}\right.$ ). This situation is depicted in figure 1(b). On the other hand, if the indirect effect of the role model bias is weaker, i.e., if $\theta^{\prime} C<B^{\prime} V_{I}$ (case (a) in figure 1), then the learning dynamic eventually comes to rest at some point along the line of equilibria. In this case, both traits can drift along this line leading to any level of conspicuous consumption as a stable outcome. This process is, however, much more slowly than in the runaway case.

Therefore, if the preference trait's mean value, $\bar{X}_{P}$ in a population is greater than the mean value of the indicator trait, $\bar{X}_{I}$, this population is characterized as a point lying above the line of equilibrium in figure 1 . This implies that an average consumer prefers a level of consumption, $\bar{X}_{I}$, measured by $\bar{X}_{P}$, that lies above the average value of the indicator trait in the population. As a consequence, the mean value of the indicator trait increases, i.e., the level of overall consumption is rising. At the same time, the role model bias also causes the value of the preference trait to increase due to the fact that both traits are positively correlated: social learners adopt from their role models even more accentuated status preferences than they had before. In 
Veblen's words, this process “...will give place to a restless straining to place a wider and everwidening pecuniary interval between himself and this average standard" $(1899,31)$. The model's learning dynamics are therefore capable of generating typical Veblenian consumption dynamics. The learning biases in cultural transmission, taken together, produce such a self-augmenting "treadmill" of consumption cycles.

We now drop the requirement that $\frac{H-\bar{X}_{I}}{S}=0$ to gain further insights into the model's learning dynamics. By doing so, we account for an environment that favors particular variants of the indicator trait. The parameter $S$ measures the intensity of the "selection" of cultural role models in a certain environment $H$. By iterating the recursions for $\bar{X}_{I}$ and $\bar{X}_{P}$, given by the equations (13) and (14), for many social learning steps, we gain some additional insights on the underlying learning dynamics. Figure 2 shows the development of the indicator and preference traits in time for different parameter values.
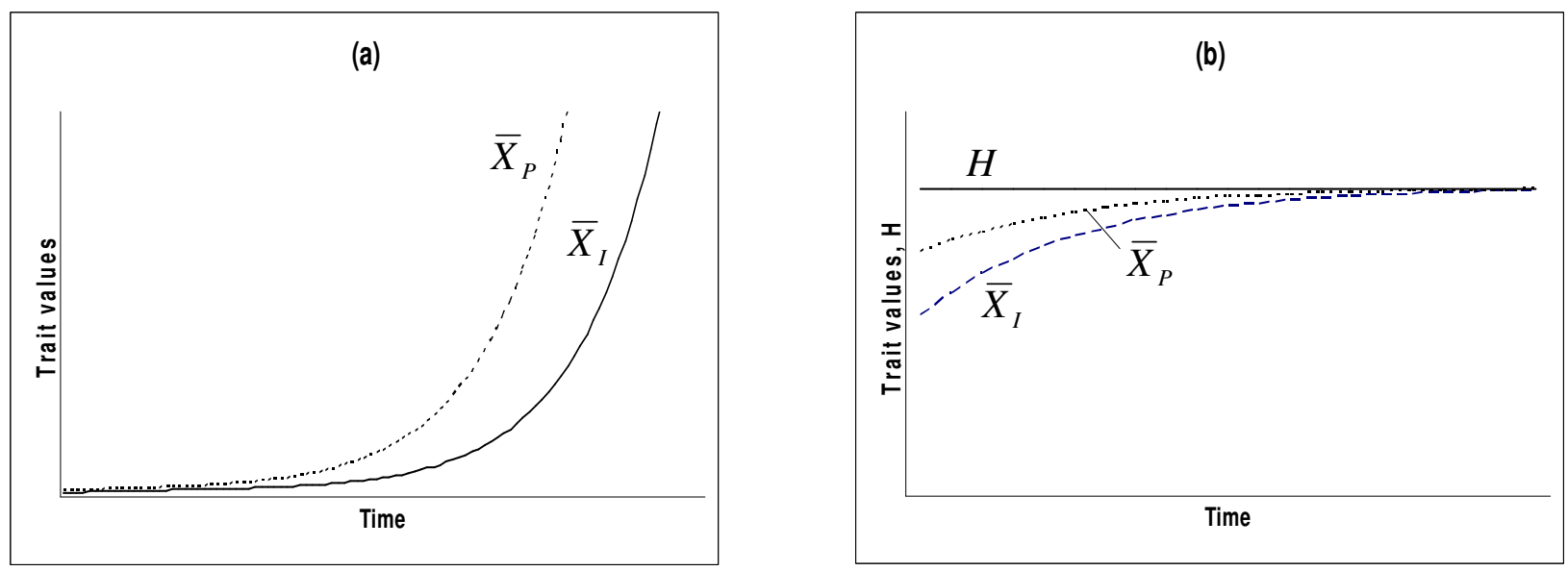

Figure 2: The development of $\bar{X}_{I}$ and $\bar{X}_{P}$ in time given that the strength of $\theta^{\prime}$ is greater than the combined influence of $S$ and $B^{\prime}$ in case (a) and smaller in case (b).

Figure 2 (a) shows that if the strength of the role model bias is strong compared to the combined forces of "selection" and direct bias, then the values of both the indicator trait and the preference trait will still run away. In this case, biased cultural transmission is stronger than the influence of an environment that favors, for example, a certain consumption behavior. Certainly, this process cannot continue forever; some factors will eventually restrain this dynamic. In the long-run, an increase in consumption activities may, for example, face ecological constraints. Hence, some constraints not accounted for in the model will eventually limit the evolution of the cultural traits in the population. If the strength of indirect bias is weak compared to the combined forces of the environment and direct bias, then both traits will evolve so that at equilibrium naïve individuals tend to imitate models with the optimal value of the indicator trait as it is shown in figure 2 (b).

If $\bar{X}_{I}=H$ (implying that also $\bar{X}_{P}=H$ ), the mean value of the indicator trait is the value of the highest cultural influence. Thus, the environment will cause cultural transmission to be biased toward the cultural variant that maximizes cultural influence. However, this equilibrium is unstable if the influence of the environment and direct bias on the indicator trait together are less 
than the strength of indirect bias on the preference trait. In this case, they will both again "run away". If this is not the case, the equilibrium is stable and the indicator trait converges to the optimum from any initial condition, i.e., the average individual finds agents with the optimum value of the indicator trait most attractive.

There may also be a "drift-away" process, in which case every point on the line of equilibrium is neutrally stable and the population "drifts" along this line. This process allows any value of the indicator trait to become common in the population, even if it is "selected" against. This implies that different groups of social learners living in the same environment $H$ will exhibit different levels of conspicuous consumption. In this case, the environment's influence and direct bias are balanced by the force of indirect bias. Even if, for example, a certain consumption pattern is highly disadvantageous for the agents showing it, a strong role model bias enables it to spread within the population of social learners.

\section{The cultural and biological determinants of human consumption behavior}

The model of cultural evolution presented in the last section represents a way to distinguish behaviors whose evolutionary dynamics are primarily genetic from those whose dynamics are primarily cultural. Moreover, it bears no resemblance to genetic models other than both being about evolutionary processes. The specific dynamics of cultural learning are rather unique. The crucial differences between evolutionary rates and equilibria of biological and cultural traits are to be explained by differences in the underlying modes of transmission. After having made some appropriate changes in this respect, much of the conceptual framework of population genetics can usefully be extended to cultural evolution to account for these differences (see Cavalli-Sforza and Feldman, 1981). Such models direct our attention to cultural learning phenomena we are unlikely to consider if we stick to analogy or metaphor constructions from biology as focusing devices for theory development in economics. This section sheds some light on the ontological relations between the biological and cultural domains.

\section{The origins of human learning capacities}

Social learning is the basis of culture. The underlying human learning mechanisms including the innate aspects of cognitive learning biases - were crafted by natural selection in a Darwinian understanding. Therefore, Darwinian concepts are pivotal to an understanding of the evolutionary origins of these psychological capacities that give rise to cultural evolution (see Tomasello, 1999; Singer, 2000). The learning biases, arising from human psychology, are one reason why cultural evolution produces outcomes different form those in genetic evolution. In our model above, we introduced an indirect or role model bias: people are driven to emulate others, especially those believed to be of higher status. Such prestige-biased cultural transmission occurs when individuals choose models based on indicators of prestige or status. Humans pay particular attention to, preferentially interact with, and tend to imitate successful or prestigious individuals (see Henrich and Gil-White, 2001). As to its evolutionary origins that are explicable by Darwinian principles of natural selection, it has been argued that selection favored social learners who have been able to evaluate potential models and copy the most successful among them, thereby saving the costs of individual learning (see also Rogers, 1983; Labov, 2001). This method of evaluating different cultural variants is likely to be much less costly than directly

evaluating these variants (Boyd and Richerson, 1985, 135). In addition, the formal model contained a direct or content-based bias: there are cognitive structures that cause people to preferentially adopt some cultural variants rather than others. Many examples can be given for 
such evolved direct cognitive biases: people show, for example, an inclination toward cooperative behavior (e.g., Rubin, 1982; Fehr and Schmidt, 1999; Cordes et al., 2007), a systematic bias in favor of materialism (see $\mathrm{Ng}, 2003$ ), or instinctively prefer status-signaling goods (Veblen, 1899). Also direct or content-based biases can be accounted for by referring to their evolutionary past, i.e., they are a result of biological evolution in an ancient environment.

Content-dependent and prestige- or status-based psychological learning biases affect together with other transmission biases (see Richerson and Boyd, 2005, 69) - the spread of cultural representations. Hence, for an analysis of cultural evolution, we need to understand how cognition directs social learning toward certain individuals or cultural contents. Moreover, we need to understand how cognition makes use of socially available information in a population (Henrich et al, 2007). Culture has a much richer array of cognitive dispositions that take effect at the population level than is the case for genes or gene-like entities. Hence, innate cognitive structures shape the human mind and are of great importance for understanding human culture. They interact with social processes and the cognition of social learning to influence the dissemination of cultural variants and the associated behavioral products. At every instance of cultural transmission from one individual to another, cognitive and emotional learning biases affect the mental representations of cultural variants (see Norenzayan and Heine, 2005). A naturalistic approach seeks to account for the psychological underpinnings of these biases. It is here that continuities in ontology between the cultural and the biological domains are to be found.

\section{The role of "replicators" in cultural and biological evolution}

Except for rare mutations, a gene is a faithful copy of a single gene carried by a member of the previous generation. Moreover, each gene is equally likely to be included in an individual's gametes (Henrich and Boyd, 2002). Therefore, genetic replication is - in contrast to cultural transmission - unbiased. Cultural variants, however, are not transmitted intact from one brain to another (see Sperber, 1998; Boyer, 1999). This implies that cultural contents undergo some sort of modification during communication (Atran, 2001). In addition, cultural contents just give rise to observable behaviors and imitating agents must then infer the underlying mental representations. There are inferential transformations between observed behavior and mental cultural representations formed by an individual. This process may be highly inaccurate. ${ }^{1}$ Cultural variants can cross and merge so quickly that there can be no identification of "lineages". What is more, in humans, genetic transmission is strictly vertical. This implies that there is no horizontal or oblique passing on of information between the agents, as it is the case in cultural transmission (see Cavalli-Sforza and Feldman, 1981).

The culturally transmitted characters in the model above - the indicator trait $\bar{X}_{I}$ and the preference trait $\bar{X}_{P}$ - have a continuous range of values, which is different from the mostly dichotomous genetic transmission. ${ }^{2}$ They are blended entities that never exactly replicate. In our model, this is mirrored by the blending of cultural variants via the sampling of cultural role models. Blending, in combination with indirectly biased transmission, gives rise to mental representations that are not represented among the $n$ individuals sampled $\left(Z_{I 1}, \ldots, Z_{I n}\right)$, i.e., there is no replication of existing mental representations. But still, this dynamic preserves variation and

\footnotetext{
${ }^{1}$ Continuous, non-discrete, trait models allow for substantial error and other forms of non-replication.

2 There are some blended traits in genetic transmission as well, such as body size. However, also continuous and blended genetic traits must survive transmission with reliably measurable frequency and fidelity to produce "selectable" differences (Atran, 2001). In contrast to cultural evolution, selectable traits must in themselves be cohesive and coherent proportions.
} 
causes an evolving cultural system. Even if cultural transmission is inaccurate at the level of individuals, it does not follow there can be no cumulative evolution or, for example, cultural inertia. Conformist transmission, for example, leads to cultural inertia at the level of the population (see, e.g., Aronson et al., 2002, ch. 8; Kameda and Diasuke, 2002). Imitation and other forms of social learning do not need to be highly accurate to enable culture to evolve in a meaningful way (McElreath and Henrich, 2007). Therefore, the fact that culture evolves does not imply that there are units analogous to genes. Boyd and Richerson (1985) present a variety of models of cultural evolution that prove this point.

Human learning abilities are constrained and biased in many ways (Rogers, 1988; Richerson and Boyd, 2005). The human mind's propensity to preferentially focus attention on certain individuals or contents implies that the normal approach to replication of cultural entities analogous to genetic reproduction is necessarily insufficient (see Henrich et al., 2007). The specific details of the underlying cultural dynamics can only be understood by taking into account the concrete social, psychological, and ecological processes involved. An application of abstract meta-theoretical approaches derived form biology, such as a generalized Darwinism, do not live up to these complex, domain-specific causal mechanisms of cultural evolution.

\section{Selection processes and recursive relations}

As a further implication of the unique causal mechanisms of cultural evolution, there is no "frictionless" adaptation as in genetic or gene-like replication, where accurate, unbiased replication allows selective forces to generate and preserve adaptations (see Henrich et al., 2007). One reason is that there are close interactions between the levels and entities of analysis in our formal model: the environment - represented by the role models $\left(Z_{I 1}, \ldots, Z_{I n}\right)$ and the populationlevel distribution of cultural variants among those (represented by $\bar{X}_{I}$ and $\bar{X}_{P}$ ) - interacts directly with the values of the indicator and preference traits on an individual level in each instance of social learning. Hence, individual characteristics and the environment are coevolving on much shorter time scales than any biological entities and driven by psychological processes, not demographic events. This close recursive relationship in combination with blended inheritance blurs an essential attribute of any selection argument (for details see Cordes, 2006): the stability of selective characteristics and environment over time. A prerequisite for natural selection to produce systematic change is a certain degree of inertia on the part of the environment and the unit of selection. Consequently, a fundamental component of any theory of inheritance and selection - which a generalized Darwinism embraces - is insistence on the temporary constancy of the genetic material or any equivalent to enable selection forces to work.

In contrast, the environment of cultural and economic systems is characterized by many variables changing simultaneously - as has been demonstrated in the model above - preventing something like natural selection forces to work in a systematic way, i.e., to direct the distribution of cultural traits in a population in a certain direction (see also Witt, 2004). Another problem relates to the question of what the units of selection are in this context. There are no constant units of selection in our blending model. In the course of cultural transmission, inferential processes on the part of the communicating agents transform the cultural variants (Henrich and Boyd, 2002). These modification processes during transmission are more important in the cultural sphere than any selection-like process as regards the generation of cultural change. Cognitive biases, for example, lead to cultural change.

When analyzing cultural evolution, we have to take into account the fact that there is indeed a systematic and direct feedback between entities and levels of analysis that restricts the explanatory power of any analogy construction to the biological domain. The model has shown 
that "selective" forces in the cultural realm - together with cultural learning biases - lead to outcomes that are qualitatively different from processes interpreted analogously to natural selection in biological evolution. Therefore, it is problematic to talk about "natural selection" in the cultural sphere, as it is done by, for example, Knudsen (2002), who uses the term "economic natural selection". The relative rates of competing evolutionary forces, the underlying causal mechanisms, and their qualitative results are very different in these two realms (see also McElreath and Henrich, 2007). That is the reason why a generalized Darwinism cannot account for the ontological continuities between the biological and cultural domains.

\section{The different notions of "fitness"}

The recursions for the learning dynamic derived above can explain some facets of human behavior that are hard to understand from a sociobiological point of view. The cultural process in our model is driven by naïve individuals choosing models based on the value of the indicator trait. Humans are selective in picking the individuals they will learn from. For instance, they preferentially learn from successful or prestigious people. Therefore, the "fitness" of a cultural variant will depend jointly on how attractive its content is and how it affects an individual's likelihood of being chosen as a role model by other individuals. If these individuals choose role models based on a character marking, for example, prestige or status, cultural evolution easily enables otherwise maladaptive traits to spread; less admired agents showing better adapted behaviors in a certain environment are then less likely to be imitated (Boyd and Richerson, 1985, 260). In our model, cultural learning causes the indicator trait to coevolve with the preference trait so as to cause the indicator trait, for example, a certain consumption pattern, to take on exaggerated forms. We have seen that a prestige-biased force can lead to "runaway" cultural evolution (Richerson and Boyd, 2005, 163-4). For this process to happen there need not be any adaptive explanation; aspects of human behavior, such as the maladaptive display of prestige, become understandable in terms of indirect bias (see Boyd and Richerson, 1985, 259). Cognitive dispositions evolved by natural selection may impose significant constraints on some human behaviors, but negligible constraints on others.

What, then, are the causal mechanisms behind the processes that bestow different "fitnesses" on cultural variants? If the only criterion is their successful spreading in a population, then the concept of selection ends up in a misleading tautology (e.g., Henrich et al., 2007). It is hard to imagine something like a "cultural fitness" of mental representations inferred from their success transmission through the population of social learners alone. Evolutionary biologists do not face this problem for they are typically concerned with concrete cases of selection and the underlying causal links. In a similar manner, to avoid tautological avenues, economists should be concerned with concrete processes of cultural learning and individual behavior as well as with their underlying domain-specific causal mechanisms to explain real world phenomena.

In the case of humans, natural selection has produced mechanisms of cultural transmission that are neither necessarily adaptive nor consistent with the predictions of acultural, biological evolutionary models, i.e., models that do not include cultural evolution (see Boyd and Richerson, 1985; Rogers, 1988; Richerson and Boyd, 2005). The model of cultural evolution presented in the last section allows for the spread of "maladaptive" culturally transmitted behaviors: the role model bias evolved as a heuristic for choosing among different cultural variants in order to find out the most appropriate behavior in a certain environment. In some cases, however, indicator traits that were initially correlated with success become exaggerated. A similar dynamic, Veblen pointed out, underlies the evolution of good manners and etiquette. They started as a way to show good will and status. However, good manners and etiquette then evolved into a means to 
"...show that much time has been spent in acquiring them" $(1899,47)$. If an agent knows how to behave according to these rules and habits, this is an indicator of wealth for it implies that the individual has the leisure to learn these conventions. This process includes the potential to lead to exaggerated forms of cultural behavior.

Hence, the behaviors resulting from biased cultural transmission need not be consistent with Darwinian processes, although these gave rise to the underlying learning capabilities and learning biases. A genetically evolved system for acquiring behavior via cultural learning - establishing the ontological continuity between the domains - gives rise to equilibria that are qualitatively different from those the genes themselves would be selected to arrive at (see McElreath and Henrich, 2007). Natural selection has influenced cultural evolution during a period of coevolution and often has produced cultural fitnesses that are close analogs to genetic fitnesses. The underlying causal mechanisms, however, are fundamentally different from those underlying psychological processes in cultural evolution. It is unlikely that an approach based on some abstract Darwinian principles derived from the causal structure of biological evolution is capable of grasping the subtleties of these mechanisms of cultural evolution.

\section{Conclusions}

"Despite his immense emphasis on Darwinism, Veblen never systematically applied to social or economic phenomena the leading Darwinian concepts of natural selection, survival of the fittest, struggle for existence, and adaptation." (Davis, 1945, 137)

The causal mechanisms that are important for understanding cultural evolution are not the same that are important for understanding genetic evolution or abstract systems derived from it. As has been demonstrated in this paper, population-based models of cultural evolution can be useful to bring out these differences (see also Henrich et al., 2007). This approach provides the analytical apparatus for grasping cultural transmission. Biology alone does not offer an accurate analytical basis for understanding cultural evolution. Consequently, evolutionary processes that do not invoke explanations based on the causal mechanisms of genetic evolution can shed light on why and how cultural dynamics emerge. To show this, we devised a model of Veblen's conspicuous consumption. Veblen himself did not seek an analogy from biology for cultural theorizing and had a very limited interest in, for example, selection processes in the cultural realm (Jennings and Waller, 1998). Furthermore, such an endeavor would have been inconsistent with his main theoretical contributions, for example, his explanation of cumulative institutional change (see Cordes, 2007a).

Many social scientists think too narrowly in terms of, for example, the gene metaphor (see McElreath and Henrich, 2007). We argue here that it is more productive for theory development in economics to drop the genetic analogy and instead study cultural transmission and evolution on its own. For instance, cultural evolutionary models do not rely on "particulate entities" (see Boyd and Richerson, 1985, 70-80). Unlike gene-like entities, cultural representations are rarely discrete units and are not replicated during cultural transmission (Henrich and Boyd, 2002). Cultural transmission processes are - compared to genetic systems - incomplete and imperfect. Therefore, accurate replication rarely occurs. ${ }^{1}$ For that reason, the notion of a "replicator" in the cultural domain is problematic. Furthermore, as has been shown by means of a formal model, gene-like replicators are not necessary for cumulative cultural evolution to happen and mental

\footnotetext{
${ }^{1}$ It is hard to imagine a "generative replicator" carrying developmental information that can even "turn input signals from an environment into developmental instructions" in the cultural domain as has been suggested by Hodgson and Knudsen (2007).
} 
representations need not to be discrete entities to enable culture to evolve in a meaningful way. It is not necessary to assume gene-like replicators exist to deploy these models of cultural evolution. In addition, nonadaptive behaviors can spread in a population under the influence of learning biases taking effect in cultural transmission, even when they face something like "selection" that favors more adaptive behaviors in a certain environment. Moreover, instable cultural learning dynamics can arise from the genetically adaptive uses of learning heuristics, such as a role model bias (Boyd and Richerson, 1985, 279).

Differences in the underlying causal mechanisms between biological phenomena and human cultural behavior are significant and suggest that there is no basis for supposing analogous parallels or generalized principles between these domains. The abstract principles of variation, inheritance, and selection do not enable a better understanding of how cultural evolution works. It is unclear how these principles could be applied to explain real-world phenomena. Darwinism, however, is an empirically oriented scientific approach, so that the principles of variation, inheritance, and selection must have empirical applicability (see Poirot, 2007). Generalized Darwinism still needs to proof that this is possible in the cultural sphere.

A comprehensive theory of human behavior would incorporate both genetic and cultural evolution as well as their ontological continuities, while accounting for the fact that the dynamics and underlying causal mechanisms of evolution differ in these realms. Scientific progress takes place through a combination of empirical research and innovative changes in conceptual thinking. However, in how far meta-theories such as a generalized Darwinism can be fruitful in this respect is in doubt. The aim should rather be to devise theories of cultural evolution that take into account the actual properties of the cultural and economic system.

\section{References}

Aronson, Elliot, Wilson, Timothy D. and Akert, Robin M. (2002): Social Psychology, Upper Saddle River, NJ: Prentice-Hall.

Atran, Scott (2001): "The Trouble with Memes: Inference versus Imitation in Cultural Creation", Human Nature, Vol. 12, No. 4, pp. 351-381.

Becker, Markus C., Lazaric, Nathalie, Nelson, Richard R. and Winter, Sidney G. (2005): "Applying Organizational Routines in Understanding Organizational Change", Industrial and Corporate Change, Vol. 14, No. 5, pp. 775-791.

Boyd, Robert and Richerson, Peter J. (1985): Culture and the Evolutionary Process, Chicago: University of Chicago Press.

Boyer, Pascal (1999): "Cognitive Tracks of Cultural Inheritance: How Evolved Intuitive Ontology Governs Cultural Transmission", American Anthropologist, Vol. 100, No. 4, pp. 876-889.

Campbell, Donald T. (1965): "Variation and Selective Retention in Socio-Cultural Evolution", in: Barringer, H. R., Blanksten, G. I. and Raymond, M. W. (eds.), Social Change in Developing Areas, Cambridge: Schenkman Publishing Company, pp. 19-49.

Cavalli-Sforza, Luigi L. and Feldman, Marcus W. (1981): Cultural Transmission and Evolution: A Quantitative Approach, Princeton: Princeton University Press.

Cordes, Christian (2005): "Veblen's 'Instinct of Workmanship,' its Cognitive Foundations, and Some Implications for Economic Theory", Journal of Economic Issues, Vol. 39, No. 1, pp. 1-20.

-- (2006): "Darwinism in Economics: From Analogy to Continuity", Journal of Evolutionary Economics, Vol. 16, No. 5, pp. 529-541.

-- (2007a): "Can a Generalized Darwinism Be Criticized? A Rejoinder to Geoffrey Hodgson", Journal of Economic Issues, Vol. 41, No. 1, pp. 277-281.

-- (2007b): "Turning Economics into an Evolutionary Science: Veblen, the Selection Metaphor, and Analogical Thinking", Journal of Economic Issues, Vol. 41, No. 1, pp. 135-154.

Cordes, Christian, Richerson, Peter J., McElreath, Richard and Strimling, Pontus (2007): "A Naturalistic Approach to the Theory of the Firm: The Role of Cooperation and Cultural Evolution", Journal of Economic Behavior and Organization, forthcoming 
Davis, Arthur K. (1945): "Sociological Elements in Veblen's Economic Theory", Journal of Political Economy, Vol. 53, No. 2, pp. 132-149.

Fehr, Ernst and Schmidt, Klaus M. (1999): "A Theory of Fairness, Competition, and Cooperation", Quarterly Journal of Economics, Vol. 114, pp. 817-868.

Harrington Jr., Joseph E. (1999): "Rigidity of Social Systems", Journal of Political Economy, Vol. 107, No. 1, pp. 40-64.

Henrich, Joseph and Boyd, Robert (2002): "On Modeling Cognition and Culture", Journal of Cognition and Culture, Vol. 2, No. 2, pp. 87-112.

Henrich, Joseph, Boyd, Robert, and Richerson, Peter J. (2007), Five Misunderstandings about Cultural Evolution, Manuscript.

Henrich, Joseph and Gil-White, Francisco J. (2001): "The Evolution of Prestige: Freely Conferred Deference as a Mechanism for Enhancing the Benefits of Cultural Transmission", Evolution and Human Behavior, Vol. 22, pp. 165-196.

Hodgson, Geoffrey M. (2002): "Darwinism in Economics: From Analogy to Ontology", Journal of Evolutionary Economics, Vol. 12, pp. 259-281.

Hodgson, Geoffrey M. and Knudsen, Thorbjørn (2006): "Why We Need a Generalized Darwinism, and Why Generalized Darwinism is Not Enough", Journal of Economic Behavior and Organization, Vol. 61, No. 1, pp. 1-19.

-- (2007): "Information, Complexity and Generative Replication", Biology and Philosophy, forthcoming.

Jennings, Ann and Waller, William (1998): "The Place of Biological Science in Veblen's Economics", History of Political Economy, Vol. 30, No. 2, pp. 189-217.

Kameda, Tatsuya and Diasuke, Nakanishi (2002): "Cost-benefit Analysis of Social/Cultural Learning in a Nonstationary Uncertain Environment: An Evolutionary Simulation and an Experiment with Human Subjects", Evolution and Human Behavior, Vol. 23, pp. 373-393.

Knudsen, Thorbjørn (2002): "Economic Selection Theory", Journal of Evolutionary Economics, Vol. 12, pp. $443-$ 470.

Labov, William (2001): Principles of Linguistic Change: Social Factors, Oxford: Blackwell.

McElreath, Richard and Boyd, Robert (2007): Modeling the Evolution of Social Behavior: A Guide for the Perplexed, Chicago: University of Chicago Press.

McElreath, Richard and Henrich, Joseph (2007): "Modeling Cultural Evolution", in: Dunbar, R. and Barrett, L. (eds.), Oxford Handbook of Evolutionary Psychology, New York: Oxford University Press, pp. 1-18.

Nelson, Richard R. and Winter, Sidney G. (1982): An Evolutionary Theory of Economic Change, Cambridge, Mass.: Belknap Press of Harvard University Press.

Ng, Yew-Kwang (2003): "From Preference to Happiness: Towards a More Complete Welfare Economics", Social Choice and Welfare, Vol. 20, pp. 307-350.

Norenzayan, Ara and Heine, Steven J. (2005): "Psychological Universals: What Are They and How Can We Know?", Psychological Bulletin, Vol. 131, No. 5, pp. 763-784.

Poirot, Clifford (2007): "How Can Institutional Economics be an Evolutionary Science?", Journal of Economic Issues, Vol. 41, No. 1, pp. 155-179.

Richerson, Peter J. and Boyd, Robert (2005): Not by Genes Alone: How Culture Transformed Human Evolution, Chicago: The University of Chicago Press.

Rogers, Alan R. (1988): "Does Biology Constrain Culture?", American Anthropologist, Vol. 90, No. 4, pp. 819-831.

Rogers, Everett M. (1983): Diffusion of Innovations, New York: Free Press.

Rubin, Paul H. (1982): "Evolved Ethics and Efficient Ethics", Journal of Economic Behavior and Organization, Vol. 3, No. 2-3, pp. 161-174.

Singer, Wolf (2000): "Response Synchronization: A Universal Coding Strategy for the Definition of Relations", in: Gazzaniga, M. S. (ed.), The New Cognitive Neurosciences, Cambridge, Mass.: The MIT Press, pp. 325-338.

Sperber, Dan (1998): Explaining Culture: A Naturalistic Approach, Oxford: Blackwell Publishers.

Tomasello, Michael (1999): The Cultural Origins of Human Cognition, London: Harvard University Press.

Veblen, Thorstein B. (1899): The Theory of the Leisure Class, New York: The Viking Press.

Vromen, Jack (2004): "Conjectural Revisionary Economic Ontology: Outline of an Ambitious Research Agenda for Evolutionary Economics", Journal of Economic Methodology, Vol. 11, No. 2, pp. 213-247.

Witt, Ulrich (2001): "Learning to Consume - A Theory of Wants and the Growth of Demand", Journal of Evolutionary Economics, Vol. 11, pp. 23-36.

-- (2003): The Evolving Economy - Essays on the Evolutionary Approach to Economics, Cheltenham: Edward Elgar.

-- (2004): "On the Proper Interpretation of 'Evolution' in Economics and its Implications for Production Theory", Journal of Economic Methodology, Vol. 11, No. 2, pp. 125-146. 